\title{
Photopumped laser oscillation and charge-injected luminescence from organic semiconductor single crystals of a thiophene/phenylene co-oligomer
}

\author{
Musubu Ichikawa, ${ }^{a}$ Kiyoshi Nakamura, Masamitsu Inoue, Hiromi Mishima, \\ Takeshi Haritani, Ryota Hibino, Toshiki Koyama, and Yoshio Taniguchi \\ Department of Functional Polymer Science, Faculty of Textile Science and Technology, Shinshu University, \\ Tokita 3-15-1, Ueda 386-8567, Japan
}

(Received 22 June 2005; accepted 4 October 2005; published online 23 November 2005)

\begin{abstract}
We have demonstrated that single crystals of a thiophene/phenylene co-oligomer $[\alpha, \omega$-bis-biphenyl-4-yl-terthiophene (BP3T) $]$ show interesting photonic aspects: (1) the self-waveguided amplified spontaneous light emissions with a comparable low threshold of $8 \mu \mathrm{J} / \mathrm{cm}^{2}$ to other optimized organic solid-state laser systems, and (2) the laser oscillation based on the optical self-confinement effect in the crystals. We have also presented electroluminescence from the crystals based on bipolar injection and the crystals' tolerance for intense current driving. These achievements strongly imply that BP3T crystals are a promising candidate for organic laser diodes. (C) 2005 American Institute of Physics. [DOI: 10.1063/1.2138361]
\end{abstract}

The achievement of long-lasting, bright electroluminescence with high efficiency in both polymeric ${ }^{1}$ and lowmolecular-weight organic materials ${ }^{2}$ has inspired the development of a new class of laser diodes (LDs). Within this development, optically induced solid-state organic lasers have been proposed and fabricated based on thin films by methods such as solution casting and vacuum deposition with a wide range of related organic semiconductors. ${ }^{3-7}$

As a counterpart of these research streams, crystalline molecular materials also get much attention as promising candidates for organic LDs demanding high-density electric pumping for population inversion. Organic molecular single crystals exhibit superior charge-carrier transport properties to amorphous and polymeric organic semiconductors. ${ }^{8}$ This advantage is due to the inherent long-range structural ordering of these single crystals. Furthermore, there are several examples of stimulated emission, including amplified spontaneous emission ${ }^{9-14}$ (ASE), from molecular single crystals. These excellent photonic and electronic features imply the possibility of current-injection lasing. However, chargeinjected luminescence from organic single crystals with high stimulated emission properties has not yet been reported. We have achieved optically induced laser oscillation and chargeinjected luminescence from a molecular single crystal. This breakthrough opens up a new research path toward developing organic LDs.

The synthesis of a thiophene/phenylene co-oligomer, $\alpha, \omega$-bis-biphenyl-terthiophene (BP3T) used can be seen in our previous work. ${ }^{15}$ The procedures of the crystal growth were also described in detail elsewhere. ${ }^{16}$ The charged pressure of the $\mathrm{N}_{2}+\mathrm{H}_{2}(10 \mathrm{~mol} \%)$ growth gas was $-18.6 \mathrm{kPa}$ (a differential pressure from the atmosphere) at room temperature. The temperature of the growth cylinder was maintained at $360{ }^{\circ} \mathrm{C}$ at the material-loaded position (bottom) and $300{ }^{\circ} \mathrm{C}$ at the growth position (top) during crystal growth (about $150 \mathrm{~h}$ ). The melting temperature of BP3T is $388^{\circ} \mathrm{C}$. The source materials of the crystals were highly purified by sublimation in advance. A $\mathrm{N}_{2}$-gas laser that generated a light

\footnotetext{
a) Author to whom correspondence should be addressed; electronic mail:
} musubu@giptc.shinshu-u.ac.jp pulse of 500 ps duration at $337 \mathrm{~nm}$ was used as an excitation light source for the gain-narrowing experiment. The detailed experimental setup was described in our previous work. ${ }^{16}$

Fabrication and measurement of the charge-injected luminescence device were carried out follows. A gold electrode that worked as an anode was thermally deposited in the thickness of $15 \mathrm{~nm}$ through a shadow mask on a polished glass substrate. Referring to our previous work, ${ }^{17}$ the thickness of the gold layer was determined to balance electric conductivity and adhesivity of crystals to both the glass substrate and gold electrode. A selected single crystal, of thickness below $1 \mu \mathrm{m}$, was placed on the gold electrode, and then the crystal spontaneously adhered to the electrode and substrate. Finally, another electrode as a cathode of $\mathrm{Au}, \mathrm{Al}$, or $\mathrm{Li}$ was thermally deposited on the fixed crystal through a shadow mask. For Li, the thickness of the metal layer was 10 $\mathrm{nm}$, and then a 200-nm-thick Al layer was simultaneously deposited on the Li layer for passivating the active $\mathrm{Li}$ under ambient conditions. For Au and Al, a 200-nm-thick layer of the metals was used as the upper electrode. The currentvoltage characteristics of the devices were measured under a vacuum $\left(10^{-3} \mathrm{~Pa}\right.$ or less $)$ at room temperature. The electroluminescence (EL) spectra were measured with a spectrometer (Ocean Optics, USB2000).

We took an optical photograph [Fig. 1(a)] and a scanning electron microscope image [Fig. 1(b)] of BP3T, thin-platelike crystals prepared with the vapor phase growth method using buffer gas. The figure also shows the chemical structure of BP3T [the inset of Fig. 1(a)]. As the optical photograph shows, the BP3T crystal is a smooth-surfaced single platelet about $1 \times 1 \mathrm{~mm}^{2}$. Fluorescence was observed only at the crystal edges except for a couple of attached small crystals. The micrograph observations of the crystals under crossed Nicols configuration indicate that bright- and darkfield images are each switched at $45^{\circ}$ rotation, indicating that the grown crystals are formed with single domain only. That is, the crystals are single crystalline. The electron microscope image [Fig. 1(b)] shows that the surface of the crystal is smooth and flat. The uniform flatness of the crystals provides optical confinements and slab-mode propagation in crystal 

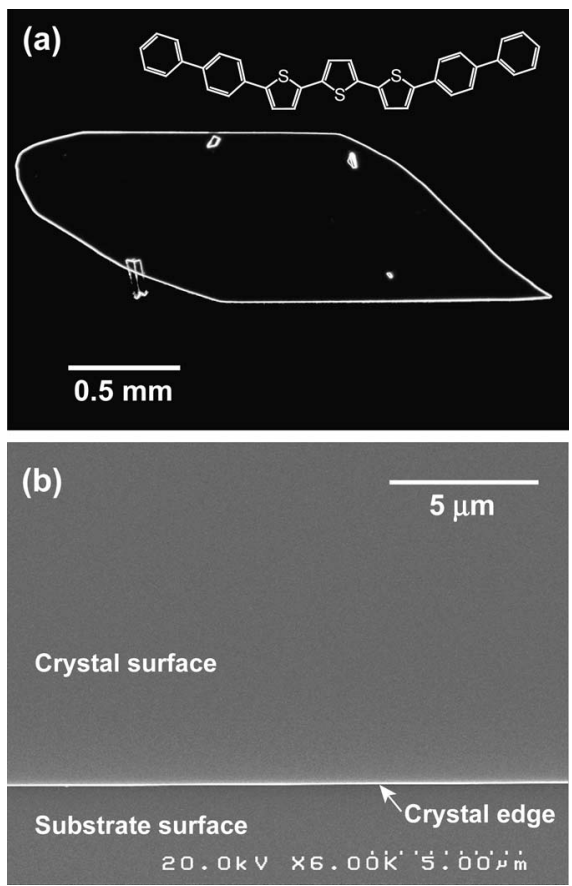

FIG. 1. (a) Optical photograph of a BP3T thin-plate-like crystal (taken under a dark field with a UV light illumination because the thinness of the crystal prevented photographing it under a bright field). Inset structure of BP3T. (b) Scanning electron microscope image of the crystal.

and highly electronic contacts with physical adhesions for fabrication of electricity-driven devices.

Figure 2(a) shows the photoluminescence (PL) spectra of BP3T thin-plate-like crystals under weak and intense excitation. The PL spectra narrowed under intense excitation. As plotted in Fig. 3, PL peak intensity increased nonlinearly above excitation laser intensity of $8 \mu \mathrm{J} / \mathrm{cm}^{2}$, and the spectral width [full width at half maximum (FWHM)] abruptly decreased around the same excitation intensity. These spectral behaviors confirm that the narrowed PL spectra results from gain narrowing, and the threshold for stimulated emission is only $8 \mu \mathrm{J} / \mathrm{cm}^{2}$. This threshold is comparable to that of other optimized organic laser systems, such as DCM2:Alq (Ref. 18) and DSB:TPD. ${ }^{19}$ The photoemission was highly
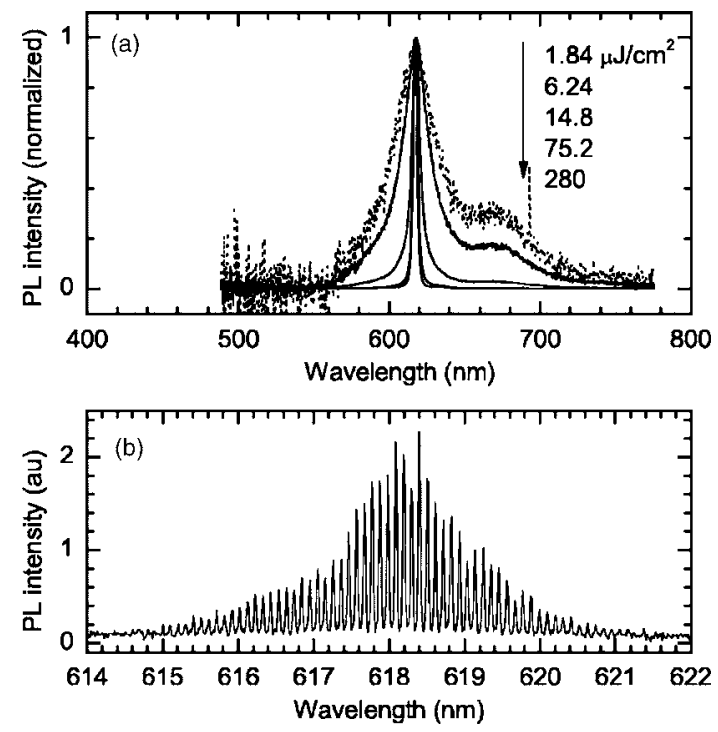

FIG. 2. (a) Laser-induced PL spectra of BP3T single crystals as a function of incident laser power density. (b) Laser oscillation spectrum of BP3T crystal with a pair of parallel facets that worked as an optical resonator at

the pumping light intensity of $130 \mu \mathrm{J} / \mathrm{cm}^{2}$
Downloaded 25 Jan 2006 to 160.252 .154 .201 . Redistribution subject to AIP license or copyright, see http://apl.aip.org/apl/copyright.jsp

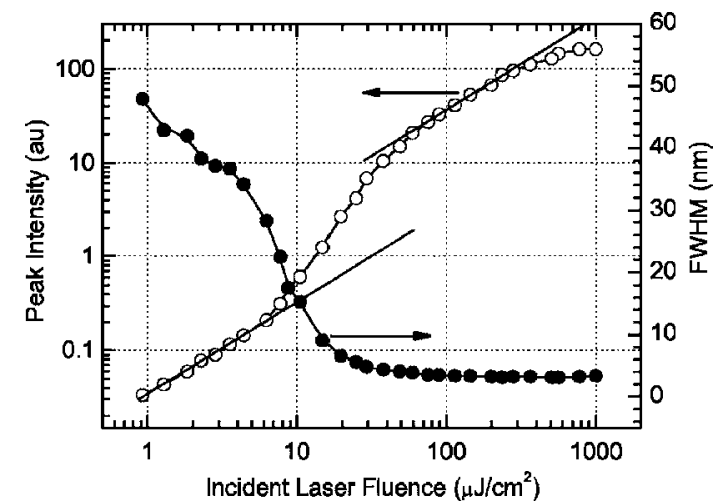

FIG. 3. Photopumping intensity dependence of peak intensities $(O)$ and FWHM values $(-)$ of PL spectra of BP3T crystals.

directional and exhibited superior output stability after thousands of shots with the excitation pulse laser. The ASE wavelength of $618 \mathrm{~nm}$ agrees with that of the second vibronic peak (0-1 transition) of the PL spectrum of the BP3T crystal under weak excitation, since the weak shoulder observed at around $580 \mathrm{~nm}$ corresponds to the first vibronic peak of the luminescence. Spontaneous emissions (i.e., PL in the weak excitation regime) from conjugated materials are usually accompanied by vibronic peaks where the emission intensities are maximal, producing the largest gain. ${ }^{5}$ The relevant vibronic levels are thus sufficient for population inversion in stimulated emission. Thus, BP3T crystals are one of the promising candidates for organic solid-state laser and organic laser diodes. The nonsaturated output of their PL peak intensity until the excitation fluence of $400 \mu \mathrm{J} / \mathrm{cm}^{2}$ is a particularly useful characteristic.

Figure 2(b) shows the laser oscillation photoluminescence spectrum with high wavelength resolution under intense excitation from another BP3T crystal that has a pair of parallel naturally formed facets that worked as a Fabry-Pérot optical resonator. The spectrum has regularly ordered progressive peaks whose interval and FWHM are 104 and 38 $\mathrm{pm}$, respectively. In addition, the center wavelength of the progressive peaks of about $618 \mathrm{~nm}$ agrees with the ASE wavelength. These features strongly indicate that the selfcavity laser oscillation occurs in multimode, a result that agrees well with our recent report on optical pumped selfcavity lasing on crystals of another oligothiophene derivative. ${ }^{20}$ Thus, the BP3T crystals confined enough photoluminescent light, and loss caused by absorption and scattering in the crystal and at its surfaces was sufficiently small. Therefore, BP3T crystals work as a self-cavity lasing medium.

Figure 4 shows the current density versus the applied electric field $(J-E)$ characteristics of the charge-injected luminescent devices using BP3T single crystals, of which the device structure was glass substrate/Au (anode)/BP3T crystal/cathode ( $\mathrm{Au}, \mathrm{Al}$, or $\mathrm{Li}$ ), and where the electric field is defined as a ratio of the applied voltage to the thickness of the crystal used. As shown in the figure, the Au cathode device shows a reversible $J-E$ characteristic as the reflection of the symmetric device structure. This reversible characteristics strongly indicates that the electric junction between the under-Au electrode and the BP3T crystal, which was fabricated by a simple procedure described before, works as well as a metal deposited junction. Some difference was observed between hole injection characteristics of adhesion electrodes 


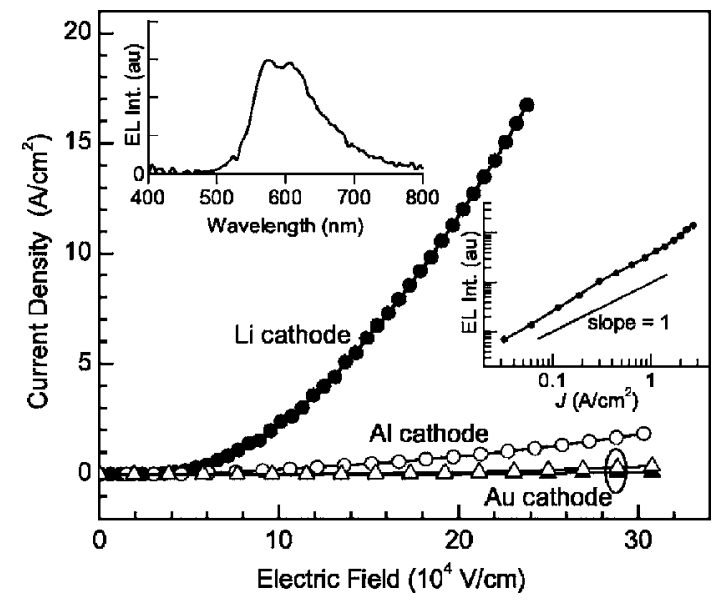

FIG. 4. J-E characteristics of several BP3T single crystal devices with different metal cathodes. The two characteristics for the Au cathode device are shown; open triangles represent the positive biased characteristics for the deposited Au cathode, while closed triangles represent those of the adhesion Au anode. Inset (upper left): EL spectrum of BP3T single crystal device. Inset (lower right): Double logarithmic plot of current density vs EL intensity characteristics of the BP3T single crystal device with a Li cathode.

and the thermally deposited electrodes. The effective contact area may have caused the difference.

As shown in Fig. 4, the cathode metal used strongly affects the $J-E$ characteristics. Since the current increased with lower work function metals, the electron injection from the cathode increases the current due to the lower Schottky barrier between the cathode and the BP3T crystal (highest occupied molecular orbital: $5.08 \mathrm{eV}$, lowest unoccupied molecular orbital: $2.76 \mathrm{eV}$ ). Particularly, on the device with a $\mathrm{Li}$ cathode, current density was 1000 times that of the Au cathode device achieved at the same external electric field strength $(200 \mathrm{kV} / \mathrm{cm})$. A maximum current density of $15 \mathrm{~A} / \mathrm{cm}^{2}$ was achieved under continuous electric driving. Such continuous high density current leads to catastrophic degradation of amorphous organic thin film used for conventional organic light-emitting diodes (OLEDs). The BP3T crystal's tolerance for intense current driving is one of its advantages for realization of an organic laser diode demanding high-density current excitation.

Moreover, the Al- and Li-cathode devices show EL at the applied voltage of only $5 \mathrm{~V}$, which is similar to conventional thin film OLEDs. The EL spectrum indicated in the upper left inset of Fig. 4 shows three vibronic progressions peaking at 572 and $614 \mathrm{~nm}$ and shouldering at around 670 $\mathrm{nm}$. These vibronic features were also observed in the PL of BP3T under weak excitation shown in Fig. 2(a); the weak shoulder was at around $580 \mathrm{~nm}$ and the two peaks were at 617 and $670 \mathrm{~nm}$. In addition, the EL was observed in the Aland $\mathrm{Li}$-cathode devices that exhibited bipolar charge injection. Therefore, the EL is concluded to be the recombination radiation based on bipolar (holes and electrons) charge injection into the BP3T crystal. In addition, the linear relationship between current density and EL intensity shown in the lower right inset of Fig. 4 also confirms the charge carrier recombination radiation. The observed lights were emitted more strongly from the crystal edge than from its surface. Hotta et $a l$. reported that the crystallographic $a b$ plane of thiophene/ phenylene co-oligomers (including BP3T) is parallel to the platelet face of the crystals, and the molecules align upright on the $a b$ plane: the long axis of BP3T is approximately perpendicular to the $a b$ plane. ${ }^{21}$ Since the luminescence transition dipole moment of such molecules is parallel to the Downloaded 25 Jan 2006 to 160.252.154.201. Redistribution subject long axis, luminescence is emitted around the $a b$ plane. Hence, the crystal radiates most of its EL from the edge face. In addition, since the crystals are thicker than the wavelength of the light, the crystals also worked as waveguides.

In this letter we demonstrated optoelectronic features of a BP3T single crystal. The BP3T crystals exhibited an excellent stimulated emission property; the crystals showed the self-waveguided ASEs with a comparable low threshold of $8 \mu \mathrm{J} / \mathrm{cm}^{2}$ to other optimized organic solid-state laser systems, and the crystals with naturally formed parallel facets that worked as an optical resonator showed multimode laser oscillation under optical excitation. Moreover, we also demonstrated EL from the BP3T crystals. The EL was waveguided in the crystal and emitted from the crystal edge face. In addition, BP3T crystals can endure intense electric excitation up to $15 \mathrm{~A} / \mathrm{cm}^{2}$ at unpulsed dc operation. These optoelectronic features of BP3T crystals clearly reveal that they are a promising candidate for organic LDs.

This work was supported by CLUSTER of the Japan's Ministry of Education, Culture, Sports, Science and Technology and also supported by the Ministry's 21 st Century COE program.

${ }^{1}$ J. H. Burroughes, D. D. C. Bradley, A. R. Brown, R. N. Marks, K. Mackay, R. H. Friend, P. L. Burns, and A. B. Holmes, Nature (London) 347, 539 (1990)

${ }^{2}$ C. W. Tang, S. A. VanSlyke, and C. H. Chen, J. Appl. Phys. 65, 3610 (1989).

${ }^{3}$ N. Tessler, G. J. Denton, and R. H. Friend, Nature (London) 382695 (1996).

${ }^{4}$ F. Hide, M. A. Diaz-Garcia, B. J. Schwartz, M. R. Andersson, P. Qibing, and A. J. Heeger, Science 273, 1833 (1996).

${ }^{5}$ M. D. McGehee and A. J. Heeger, Adv. Mater. (Weinheim, Ger.) 12, 1655 (2000).

${ }^{6}$ V. G. Kozlov, V. Bulovic, P. E. Burrows, and S. R. Forrest, Nature (London) 389, 362 (1997).

${ }^{7}$ V. Bulovic, V. G. Kozlov, V. B. Khalfin, and S. R. Forrest, Science 279, 553 (1998).

${ }^{8}$ For instance, a charge carrier mobility as high as $\sim 15 \mathrm{~cm}^{2} / \mathrm{V} \mathrm{s}$, exceeding that of $a-\mathrm{Si}: \mathrm{H}$ and comparable to that of polycrystalline indium-tin-oxide thin films, was reported by V. C. Sundar et al. in molecular crystals of rubrene [V. C. Sundar, J. Zaumseil, V. Podzorov, E. Menard, R. L. Willett, T. Someya, M. E. Gershenson, and J. A. Rogers, Science 303, 1644 (2004)].

${ }^{9}$ D. Fichou, S. Delysse, and J.-M. Nunzi, Adv. Mater. (Weinheim, Ger.) 9, 1178 (1997).

${ }^{10}$ F. Garnier, G. Horowitz, P. Valat, F. Kouki, and V. Wintgens, Appl. Phys. Lett. 72, 2087 (1998).

${ }^{11}$ O. S. Avanesjan, V. A. Benderskii, V. K. Brikenstein, V. L. Broude, L. I. Korshunov, A. G. Lavrushko, and I. I. Tartakovskii, Mol. Cryst. Liq. Cryst. 29, 165 (1974).

${ }^{12}$ H. J. Brouwer, V. V. Krasnikov, T. A. Pham, R. E. Gill, P. F. v. Hutten, and G. Hadziioannou, Chem. Phys. 227, 65 (1998).

${ }^{13}$ H. Yanagi, T. Ohara, and T. Morikawa, Adv. Mater. (Weinheim, Ger.) 13, 1452 (2001).

${ }^{14}$ M. Nagawa, R. Hibino, S. Hotta, H. Yanagi, M. Ichikawa, T. Koyama, and Y. Taniguchi, Appl. Phys. Lett. 80, 544 (2002).

${ }^{15}$ S. Hotta and T. Katagiri, J. Heterocycl. Chem. 40, 845 (2003).

${ }^{16}$ M. Ichikawa, R. Hibino, M. Inoue, T. Haritani, S. Hotta, T. Koyama, and Y. Taniguchi, Adv. Mater. (Weinheim, Ger.) 15, 213 (2003).

${ }^{17}$ K. Nakamura, M. Ichikawa, R. Fushiki, T. Kamikawa, M. Inoue, T. Koyama, and Y. Taniguchi, Jpn. J. Appl. Phys., Part 2 43, L100 (2004).

${ }^{18}$ M. Berggren, A. Dodabalapur, R. E. Slusher, and Z. Bao, Nature (London) 389, 466 (1997).

${ }^{19}$ M. Ichikawa, Y. Tanaka, N. Suganuma, T. Koyama, and Y. Taniguchi, Jpn. J. Appl. Phys., Part 1 42, 5590 (2003).

${ }^{20}$ M. Ichikawa, R. Hibino, M. Inoue, T. Haritani, S. Hotta, K. Araki, T. Koyama, and Y. Taniguchi, Adv. Mater. (Weinheim, Ger.) 17, 2073 (2005).

${ }^{21}$ S. Hotta, M. Goto, R. Azumi, M. Inoue, M. Ichikawa, and Y. Taniguchi, Chem. Mater. 16, 237 (2004).

to AIP license or copyright, see http://apl.aip.org/apl/copyright.jsp 